Research letter

\title{
Diagnostics of coupling between low-frequency loops in cardiovascular autonomic control in adults, newborns and mathematical model using cross-recurrence analysis
}

\author{
Yurii M. Ishbulatov ${ }^{1,2}$, Anton R. Kiselev ${ }^{2,3}$, Elena N. Mureeva ${ }^{2}$, Yulia V. Popova ${ }^{1,2}$, Alexander V. Kurbako ${ }^{3}$, \\ Vladimir I. Gridnev ${ }^{2,3}$, Boris P. Bezruchko ${ }^{1,3}$, Margarita A. Simonyan ${ }^{2}$, Ekaterina I. Borovkova ${ }^{2,3}$, \\ Olga M. Posnenkova ${ }^{1,2}$, Olga S. Panina ${ }^{2}$, Yurii V. Chernenkov ${ }^{2}$, Anatoly S. Karavaev ${ }^{1,2,3}$ \\ ${ }^{1}$ Saratov Branch of Kotelnikov Institute of Radio Engineering and Electronics of Russian Academy of Sciences, Saratov, Russia \\ ${ }^{2}$ Saratov State Medical University, Saratov, Russia \\ ${ }^{3}$ Saratov State University, Saratov, Russia
}

Received 15 July 2019, Revised 18 October 2019, Accepted 24 October 2019

(c) 2019, Ishbulatov Yu.M., Kiselev A.R., Mureeva E.N., Popova Yu.V., Kurbako A.V., Gridnev V.I., Bezruchko B.P., Simonyan M.A., Borovkova E.I., Posnenkova O.M., Panina O.S., Chernenkov Yu.V., Karavaev A.S.

(C) 2019, Russian Open Medical Journal

\begin{abstract}
The aim of study is to investigate effectiveness of cross-recurrence analysis for the detection of coupling between the loops of heart rate and vessel tone sympathetic control. The cross-recurrence analysis is applied to the interbeat intervals and photopletysmographic signals from the mathematical model of cardiovascular system and from adults and newborn children. The model is examined under the conditions of the four experiments: with fully operational autonomic control, autonomic control weakened by $40 \%$, autonomic control weakened by $70 \%$ and autonomic blockade. The coupling is most pronounced in adult subjects and the model with fully operational autonomic control, while it is absent under the autonomic blockade. Autonomic control is also not fully developed in newborn children. As the result, smaller coupling strength is expected.

The results of cross-recurrence analysis of the model and experimental data are compared with the results of coupling detection based on the analysos of phase synchronization. Synchronization index demonstrates good correlation with the coupling strength in the model and weak coupling in newborn children in relation to adult subjects. In both cases, no correlation is observed between the synchronization index and the results of cross-recurrence analysis.
\end{abstract}

Keywords: cross-recurrence analysis, coupled systems, autonomic control, mathematical modelling, cardiovascular system, newborn children.

Cite as Ishbulatov YuM, Kiselev AR, Mureeva EN, Popova YuV, Kurbako AV, Gridnev VI, Bezruchko BP, Simonyan MA, Borovkova EI, Posnenkova OM, Panina OS, Chernenkov YuV, Karavaev AS. Diagnostics of coupling between low-frequency loops in cardiovascular autonomic control in adults, newborns and mathematical model using cross-recurrence analysis. Russian Open Medical Journal 2019; 8: e0405.

Correspondence to: Yurii M. Ishbulatov. Address: Research Institute of Cardiology, 141, Chernyshevsky str., Saratov, 410028, Russia. Phone: +78452201899. E-mail: ishbulatov95@mail.ru.

\section{Introduction}

Cardiovascular diseases are the main cause of death in the developed countries, including the Russian Federation. Development of new noninvasive diagnostic techniques is important to solve this problem. Perspective direction of the study is the diagnostics of coupling between the loops of heart rate control and vessel tone sympathetic control.

Our previous studies [1-4] showed the coupling strength to be an important index of the cardiovascular health. However, the used approach of coupling detection, namely the calculation of total percent of phase synchronization, requires introduction of the phases of the signals from the sympathetic loops (signals of RR-intervals and photoplethysmogram (PPG) filtered in 0.05-0.15 $\mathrm{Hz}$ band). Introduction of the phases is highly complicated for the signals of biological origin that typically have wide spectra.
Many researchers consider the cross-recurrence analysis to be a powerful tool for the detection of weak coupling in biological systems [5-9]. This approach also does not require the introduction of phases. Therefore, we aim to test its applicability to the detection of coupling between the loops of autonomic control and compare it to the current approach.

In the present study, we use the mathematical model [10] of the cardiovascular system to compare the methods, because in the model it is possible to finely tune the activity of the autonomic control and to gradually reduce the coupling strengths. Both methods were then applied to the experimental data from healthy adults and newborns.

\section{Materials and methods}

The experimental data

We analyzed 15-minutes experimental electrocardiogram records (ECG) of five healthy adult subjects (four males and one 
female aged 20-25 years) and five healthy newborn subjects ( 3 days old). All adult subjects and the parents of newborn subjects signed a written consent. The studies were performed in accordance with the Declaration of Helsinki and approved by the local research Ethics Committee of the Saratov Research Institute of Cardiology (Saratov, Russia).

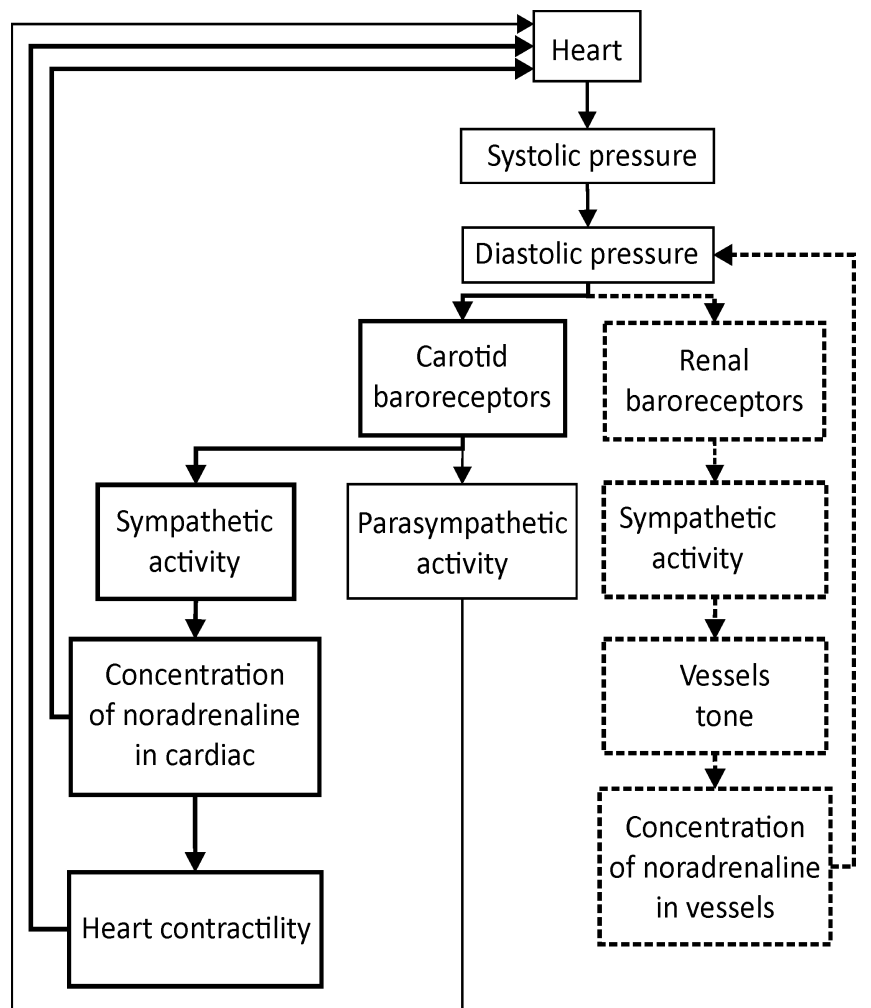

Figure 1. Structure of the mathematical model. Blocks indicated with solid bold rectangles represent the heart rate sympathetic control. Blocks indicated with thin rectangles represent the heart rate parasympathetic control. Blocks indicated with dashed bold rectangles represent the sympathetic control of the vascular tone.

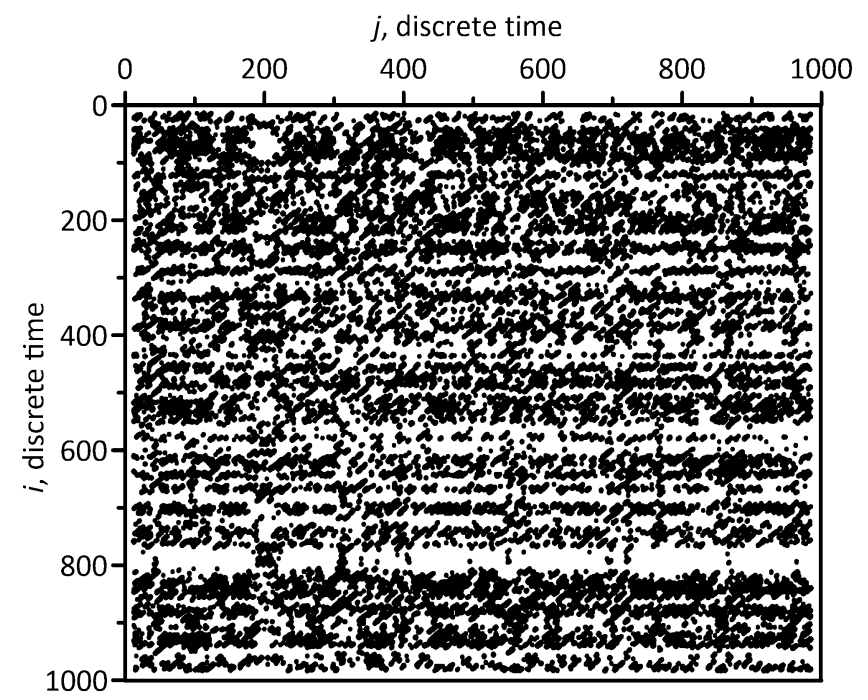

Figure 2. Cross-recurrence plot for the model interbeat intervals and PPG signals. Black dots represent close points of the two reconstructed attractors.
Experimental signals were recorded using the standard electroencephalograph analyzer EEGA-21/26 'Encephalan-131-03' (Medicom MTD Ltd, Taganrog, Russia) [http://medicom$\mathrm{mtd} . c 0 m / e n / p r o d u c t s / e e g a . h t m l]$. The signals from the adult subjects were recorded, while the subjects were resting in the quiet dimly-lit temperature-controlled room, at least 2 hours since last meal. The subjects were in supine position, were breathing spontaneously and with no forced or delayed respirations. All signals were sampled at $250 \mathrm{~Hz}$ and digitized at 14 bits resolutions.

The newborn subjects were recorded while being breast-fed to avoid motion artifacts. Both the EEG and PPG signals were recorded from the forehead.

\section{The mathematical model}

The methods were tested on the RR intervals and PPG signals from the mathematical model proposed by us in [10]. It is based on the Seidel and Herzel model [11] and Kotani model [12]. The model simulates the following processes: main heart rate, autonomic control of heart rate and heart contractility, forming of arterial pressure (AP) during the cardiac contraction and cardiac filling phases. The model also simulates the influence of the respiration on the aforementioned processes. The structure of the model is presented in Figure 1.

The main feature of the model is the two separated loops of heart rate control and vessel tone sympathetic control. The loops were modeled in accordance with the modern understanding of the nonlinear nature of autonomic control [13] and can exhibit coupling, as seen in the experimental studies [2].

The detailed description of the model is given in [10].

The model was studied under the conditions of the four numerical experiments:

- Autonomic control is fully active. All parameters of the model are chosen to achieve the best possible fit to an average healthy subject;

- Autonomic control is $60 \%$ active;

- Autonomic control is $30 \%$ active;

- Full autonomic blockade.

\section{Cross-recurrence analysis}

Cross-recurrence analysis (CRA) is the method of coupling detection between two systems. It is based on the analysis of the reconstructed attractors [8]. Taking into account the results from [14] and Takens theorem, we chose the embedding dimension $D=13$ for the reconstructed attractors of the model interbeat intervals and PPG signals.

We used the delay method to recunstruct the attractor [8]. The coordinates of the embeding space were $x(t), x(t-\tau), x(t-2 \tau)$ etc. Time delay $\tau$ was estimated as the absolute maximum of the crosscorrelation function for the model interbeat intervals and PPG signals.

Further calculations can be described with Equation 1:

$$
C R_{i, j}=\Theta\left(\varepsilon-\left\|\vec{x}_{i}-\vec{y}_{j}\right\|\right),
$$

where $\vec{x}_{i}$ and $\vec{y}_{i}$ are the points on the reconstructed HRV and PPG attractors, $i=1,2 \ldots, N, j=1,2 \ldots, N$ is the discrete time, $\Theta$ is the Heaviside function, and $\varepsilon$ is a small neighborhood. 
Then we constructed two-dimensional $N$ by $N$ plot. In accordance with eq. 1 , we calculated the distance from each point of the first attractors to every point of the second attractor. If the distance between the pair of points, for example $H R V_{i}$ and $P P G_{j}$, was less than $\varepsilon$, we set to " 1 " the point with $(i ; j)$ coordinates in the CRA plot.

Typical CRA plot for the model interbeat intervals and PPG signals is presented in Figure 2.

CRA plot, as seen in Figure 2, can be used for qualitative analysis of coupling between the interbeat intervals and PPG signals. For quantitative analysis of coupling, a number of indices can be calculated from Figure 2: average length of the diagonal lines (I); max length of the diagonal lines ( $\max I)$; Shannon entropy for the distribution of the diagonal line lengths $(E)$; average length of the vertical lines $(v)$; max length of the vertical lines $(\max v)$.

\section{The total percent of phase synchronization}

The total percent of phase synchronization ( $S$ index) was introduced in [2]. The method is based on the phase analysis approach. The instantaneous phases are introduced to the model interbeat intervals and PPG signals via the Hilbert transformation. Then the difference between the phases is calculated.

Then horisontal sections on the phase difference are automaticaly detected, they correspond to the coupling intervals. Total lengts of these intervals is calculated, then its ratio to the overal length of the signals is calculated. Percentage of this ratio is the $S$ index.

\section{Statistical analysis}

Experimental data are reported as the mean (M) with standard deviation (SD). We applied the Shapiro-Wilk test to check whether the data were approximately normally distributed. The t-test was used to compare the continuous variables between groups. The obtained estimations were considered statistically significant if $\mathrm{P}<0.05$.

Table 1. $S$ index and cross-recurrence indices calculated for the interbeat intervals and PPG signals of the model under the conditions of fully functional autonomic control, $60 \%$ functional, and $30 \%$ functional and under the autonomic blockade.

\begin{tabular}{lcccc}
\hline Index & Full control & $60 \%$ control & 30\% control & Blockade \\
\hline S index & $61 \%$ & $57 \%$ & $39 \%$ & $39 \%$ \\
$I$ & 0.55 & 0.49 & 0.55 & 0.49 \\
$\max I$ & 11.0 & 4.20 & 10.60 & 5.4 \\
$E$ & 1.15 & 0.91 & 1.13 & 0.89 \\
$V$ & 0.58 & 0.54 & 0.59 & 0.59 \\
$\max v$ & 2.40 & 2.40 & 2.40 & 2.60 \\
\hline
\end{tabular}

Table 2. $S$ index and cross-recurrence indices calculated for the interbeat intervals and PPG signals of adult subjects and newborn children.

\begin{tabular}{lccc}
\hline Index & Adults $(n=5)$ & Newborns $(n=5)$ & P-level \\
\hline Sindex, \% & $38.1 \pm 6.30$ & $19.4 \pm 6.4$ & 0.002 \\
$I$ & $0.89 \pm 0.30$ & $0.84 \pm 0.32$ & 0.805 \\
$\max I$ & $12.50 \pm 4.68$ & $9.3 \pm 8.66$ & 0.488 \\
$E$ & $1.86 \pm 0.32$ & $1.71 \pm 0.55$ & 0.612 \\
$V$ & $0.73 \pm 0.04$ & $0.78 \pm 0.01$ & 0.027 \\
$\max v$ & $2.70 \pm 0.60$ & $2.90 \pm 0.35$ & 0.538 \\
\hline
\end{tabular}

\section{Results}

The results of analysis of the model data are shown in Table 1.

As seen from Table 1, the $S$ index demonstrates monotonic decrease with the development of the autonomic blockade. This decrease is expected since the coupling between the loops of autonomic control is at maximum when the control is fully functional, and in the case of full autonomic blockade no coupling could be present. Non-zero value of the $S$ index for the model under the blockade is likely due to the presence of the dynamical noises and the badly defined instantaneous phases. The indices obtained from the cross-recurrence analysis do not agree with the dynamics of the $S$ index and do not exhibit monotonic behavior.

The results of analysis of the experimental data are shown in Table 2 in format of mean \pm standard deviation.

As seen from comparison of tables 1 and 2 the model does simulate the decreased $S$ index associated with the development of the autonomic blockade in adults and not fully developed autonomic control in children. The structure of the model also resembles the structure of the real system and our previous studies showed good qualitative accordance between the model and experimental cardiovascular data, both in health and disease [10]. It gives us the basis to interpolate the qualitative model results onto the real cardiovascular system.

From the model data we can assume that CRA indices do not reflect the dynamics of the autonomic control coupling. This result agrees well with the experimental data, since all CRA indices except for one can't distinguish adults from newborn children with the statistical significance.

The average lengths of the vertical lines ( $v$ index in table 2 ) are significantly different for adult subjects and newborn children. However, the model data suggests that it is due to the reasons unrelated to the state of autonomic control.

\section{Discussion}

Application of methods of nonlinear dynamics to the study of cardiovascular system is an important field of research [11-13, 1518], and the use of new nonlinear techniques is necessary to advance this field. However, the approbation of nonlinear techniques with the experimental data is complicated by the number of factors such as the dynamical and measurement noises, nonstationarity of the biological signals, technical and ethical limitations that restrict the design of experiments and registration of important data.

There are also problems specific to the diagnostics of coupling between the loops of autonomic control. Parameters of this system greatly vary among the patients. In [19], it was reported that the synchronization index $S$ can take the values from $30 \%$ to $50 \%$ in healthy subjects. The phenylephrine used to inflict the state of autonomic blockade also has quantitatively different effect on different patients [20]. Therefore, it is technically impossible to inhibit the activity of autonomic control by $40 \%$ and $70 \%$ in the group of patients with any degree of certainty.

To avoid the aforementioned problems, we tested the coupling detection with the cross-recurrence analysis on the mathematical model of cardiovascular system that has the structure similar to the structure of the real system [21]. Parameters of this model have a physiological sense and we can precisely set the parameters of the autonomic control of heat rate and vessel tone to simulate the stages of the autonomic blockade. 
With the model it is also possible to generate the signals of any length without the measurement noises or nonstationarity.

The mathematical model used in our study has limitations. It does not consider humoral regulation and does not take into account the hydrodynamic processes in arteries and vessels. Nonetheless, we consider it adequate for such application because in the previous studies we demonstrated the ability of the model to simulate the dynamics of $S$ index in healthy subjects and the dynamics of the cardiovascular system during the autonomic blockade [10].

In the study, the model was used to investigate the dynamics of the cross-recurrence indices during the development of the autonomic blockade and to provide better understanding of the experimental results. Only one index (average length of the vertical lines) in cross-recurrence analysis showed statistically significant difference between the adult and newborn children. However, from the model data it is evident that the $v$ index doesn't reflect the physiological processes in autonomic control, since it shows no monotonic decrease or increase with the development of the blockade.

For this reason, we think that caution should be expressed when using the cross-recurrence analysis in medical diagnostics or as a part of machine-learning algorithms.

\section{Conclusion}

The cross-recurrence analysis was applied to the detection of coupling between the autonomic control loops in the mathematical model of cardiovascular system, adult subjects and newborns. The model was studied under the conditions of four experiments: fully active autonomic control, $60 \%$ active control, $30 \%$ active control and autonomic blockade. No correlation was found between the cross-recurrence indices and the dynamics of the autonomic control. Obtained results suggest that crossrecurrence analysis is not applicable to the detection of coupling in cardiovascular system.

\section{Conflict of Interest}

The authors declare that they have no conflict of interest.

\section{Acknowledgements}

The study was funded by the President of the Russian Federation, grant MD-2202.2019.8 (registration and analysis of the data from adults) and grant MK-2492.2019.8 (registration and analysis of the data from newborns).

\section{References}

1. Prokhorov MD, Ponomarenko VI, Gridnev VI, Bodrov MB, Bespyatov $A B$. Synchronization between main rhythmic processes in the human cardiovascular system. Phys Rev E Stat Nonlin Soft Matter Phys 2003; 68(4 Pt 1): 041913. https://doi.org/10.1103/PhysRevE.68.041913.

2. Karavaev AS, Prokhorov MD, Ponomarenko VI, Kiselev AR, Gridnev VI, Ruban El, et al. Synchronization of low-frequency oscillations in the human cardiovascular system. Chaos 2009; 19(3): 033112. https://doi.org/10.1063/1.3187794.

3. Ponomarenko VI, Prokhorov MD, Bespyatov AB, Bodrov MB, Gridnev VI. Deriving main rhythms of the human cardiovascular system from the heartbeat time series and detecting their synchronization. Chaos, Solitons \& Fractals 2005; 23(4): 1429-1438. https://doi.org/10.1016/j.chaos.2004.06.041.
4. Kiselev AR, Gridnev VI, Prokhorov MD, Karavaev AS, Posnenkova OM Ponomarenko $\mathrm{VI}$, et al. Selection of optimal dose of beta-blocker treatment in myocardial infarction patients based on changes in synchronization between $0.1 \mathrm{~Hz}$ oscillations in heart rate and peripheral microcirculation. I Cardiovasc Med (Hagerstown) 2012; 13(8): 491-498. https://doi.org/10.2459/JCM.0b013e3283512199.

5. Shockley K, Butwill M, Zbilut JP, Webber CL. Cross recurrence quantification of coupled oscillators. Physics Letters A 2002; 305(1-2): 59-69. https://elibrary.ru/item.asp?id=1266163

6. González-Gómez GH, Infante O, Martínez-García P, Lerma C. Analysis of diagonals in cross recurrence plots between heart rate and systolic blood pressure during supine position and active standing in healthy adults. Chaos 2018; $28(8): \quad 085704$ https://doi.org/10.1063/1.5024685.

7. Parro VC, Valdo L. Sleep-wake detection using recurrence quantification analysis. Chaos 2018; 28(8): 085706. https://doi.org/10.1063/1.5024692.

8. Marwan N, Zou Y, Wessel N, Riedl M, Kurths J. Estimating coupling directions in the cardiorespiratory system using recurrence properties. Philos Trans A Math Phys Eng Sci 2013; 371(1997): 20110624. https://doi.org/10.1098/rsta.2011.0624.

9. Censi F, Calcagnini G, Cerutti S. Coupling patterns between spontaneous rhythms and respiration in cardiovascular variability signals. Comput Methods Programs Biomed 2002; 68(1): 37-47. https://doi.org/10.1016/s0169-2607(01)00158-4.

10. Karavaev AS, Ishbulatov JM, Ponomarenko VI, Prokhorov MD, Gridnev VI, Bezruchko BP, et al. Model of human cardiovascular system with a loop of autonomic regulation of the mean arterial pressure. J Am Soc Hypertens 2016; 10(3): 235-243. https://doi.org/10.1016/j.jash.2015.12.014.

11. Seidel $H$, Herzel $H$. Bifurcations in a nonlinear model of the baroreceptor-cardiac reflex. Physica D: Nonlinear Phenomena 1998; 115(1-2): 145-160. https://doi.org/10.1016/S0167-2789(97)00229-7.

12. Kotani K, Struzik ZR, Takamasu K, Stanley HE, Yamamoto Y. Model for complex heart rate dynamics in health and disease. Phys Rev E Stat Nonlin Soft Matter Phys 2005; 72 (4 Pt 1): 041904. https://doi.org/10.1103/PhysRevE.72.041904.

13. Ringwood JV, Malpas SC. Slow oscillations in blood pressure via a nonlinear feedback model. Am J Physiol Regul Integr Comp Physiol 2001; 280(4): R1105-R1115. https://doi.org/10.1152/ajpregu.2001.280.4.R1105.

14. Bezerianos A, Bountis T, Papaioannou G, Polydoropoulos P. Nonlinear time series analysis of electrocardiograms. Chaos 1995; 5(1): 95-101. https://doi.org/10.1063/1.166104.

15. Ernst G. Heart-rate variability - more than heart beats? Front Public Health 2017; 5: 240. https://doi.org/10.3389/fpubh.2017.00240.

16. Dimitriev DA, Saperova EV, Dimitriev AD. State anxiety and nonlinear dynamics of heart rate variability in students. PLoS One 2016; 11(1): e0146131. https://doi.org/10.1371/journal.pone.0146131.

17. Porta A, Bari V, Ranuzzi G, De Maria B, Baselli G. Assessing multiscale complexity of short heart rate variability series through a model-based linear approach. Chaos 2017; 27(9): 093901. https://doi.org/10.1063/1.4999353.

18. Bittihn P, Berg S, Parlitz U, Luther S. Emergent dynamics of spatiotemporal chaos in a heterogeneous excitable medium. Chaos 2017; 27(9): 093931. https://doi.org/10.1063/1.4999604.

19. Kiselev AR, Mironov SA, Karavaev AS, Kulminskiy DD, Skazkina VV, Borovkova El, et al. A comprehensive assessment of cardiovascular autonomic control using photoplethysmograms recorded from the earlobe and fingers. Physiol Meas 2016; 37(4): 580-595. https://doi.org/10.1088/0967-3334/37/4/580.

20. Jones PP, Shapiro LF, Keisling GA, Jordan J, Shannon JR, Quaife RA, et al. Altered autonomic support of arterial blood pressure with age in healthy men. Circulation 2001; 104(20): 2424-2419. https://doi.org/10.1161/hc4501.099308. 
21. Bezruchko BP, Smirnov DA. Extracting knowledge from time series. An introduction to nonlinear empirical modeling. Springer-Verlag Berlin Heidelberg, 2010; 410 p. https://doi.org/10.1007/978-3-642-12601-7.

Authors:

Yurii M. Ishbulatov - MS, Researcher, Laboratory of Nonlinear Dynamics Modelling, Saratov Branch of Kotelnikov Institute of Radio Engineering and Electronics of Russian Academy of Sciences, Saratov, Russia; Junior Researcher, Department of New Cardiological Informational Technologies, Research Institute of Cardiology, Saratov State Medical University, Saratov, Russia. https://orcid.org/0000-0003-2871-5465.

Anton R. Kiselev - MD, DSc, Head of Department of New Cardiological Informational Technologies, Research Institute of Cardiology, Saratov State Medical University, Saratov, Russia; Professor, Department of Nano- and Biomedical Technologies, Saratov State University, Saratov, Russia. https://orcid.org/0000-0003-3967-3950.

Elena N. Mureeva - MD, Assistant, Department of Pediatrics and Neonatology, Saratov State Medical University, Saratov, Russia. https://orcid.org/0000-0001-6065-1751.

Yulia V. Popova - MD, PhD, Senior Researcher, Laboratory of Nonlinear Dynamics Modelling, Saratov Branch of Kotelnikov Institute of Radio Engineering and Electronics of Russian Academy of Sciences, Saratov, Russia; Senior Researcher, Department of New Cardiological Informational Technologies, Research Institute of Cardiology, Saratov State Medical University, Saratov, Russia. https://orcid.org/0000-0002-2402-7588.

Alexander V. Kurbako - BS, MS student, Department of Nano- and Biomedical Technologies, Saratov State University, Saratov, Russia.

Vladimir I. Gridnev - MD, DSc, Director of Research Institute of Cardiology, Saratov State Medical University, Saratov, Russia; Professor, Department of Nano- and Biomedical Technologies, Saratov State University, Saratov, Russia. https://orcid.org/0000-0001-6807-7934.

Boris P. Bezruchko - DSc, Professor, Laboratory of Nonlinear Dynamics Modelling, Saratov Branch of Kotelnikov Institute of Radio Engineering and Electronics of Russian Academy of Sciences, Saratov, Russia; Professor, Department of Nano- and Biomedical Technologies, Saratov State University, Saratov, Russia. https://orcid.org/0000-0002-6691-8653.

Margarita A. Simonyan - MD, Junior Researcher, Department of New Cardiological Informational Technologies, Research Institute of Cardiology, Saratov State Medical University, Saratov, Russia. https://orcid.org/00000002-9866-3069.

Ekaterina I. Borovkova - MSc, Researcher, Department of New Cardiological Informational Technologies, Research Institute of Cardiology, Saratov State Medical University, Saratov, Russia; Assistant, Department of Nano- and Biomedical Technologies, Saratov State University, Saratov, Russia. https://orcid.org/0000-0002-9621-039X.

Olga M. Posnenkova - MD, DSc, Leading Researcher, Laboratory of Nonlinear Dynamics Modelling, Saratov Branch of Kotelnikov Institute of Radio Engineering and Electronics of Russian Academy of Sciences, Saratov, Russia; Head of Department of Atherosclerosis and Chronic Coronary Artery Disease, Research Institute of Cardiology, Saratov State Medical University, Saratov, Russia. https://orcid.org/0000-0001-5311-005X.

Olga S. Panina - MD, PhD, Associate Professor, Department of Pediatrics and Neonatology, Saratov State Medical University, Saratov, Russia.

Yurii V. Chernenkov - MD, DSc, Professor, Head of Department of Pediatrics and Neonatology, Saratov State Medical University, Saratov, Russia. https://orcid.org/0000-0002-6896-7563.

Anatoly S. Karavaev - PhD, Senior Researcher, Saratov Branch of Kotelnikov Institute of Radio Engineering and Electronics of Russian Academy of Sciences, Saratov, Russia; Leading Researcher, Department of New Cardiological Informational Technologies, Research Institute of Cardiology, Saratov State Medical University, Saratov, Russia; Associate Professor, Department of Nano- and Biomedical Technologies, Saratov State University, Saratov, Russia. https://orcid.org/0000-0003-4678-3648. 